\title{
Spin Splitting and Weak Localization in (110) GaAs/AlGaAs Quantum Wells
}

\author{
T. Hassenkam, S. Pedersen, K. Baklanov, A. Kristensen, C. B. Sorensen, and P. E. Lindelof \\ The Niels Bohr Institute, University of Copenhagen, Universitetsparken 5, DK-2100 Copenhagen, DENMARK \\ F. G. Pikus ${ }^{\dagger}$ \\ University of California, Santa Barbara CA 93106, USA \\ G. E. Pikus \\ A.F. Ioffe Physicotechnical Institute 194021 St Petersburg, RUSSIA
}

(April 22, 2021)

\begin{abstract}
We investigate experimentally and theoretically the spin-orbit effects on the weak localization in a (110) GaAs 2-dimensional electron gas (2DEG). We analyze the role of two different terms in the spin splitting of the conduction band: the Dresselhaus terms, which arise due to the lack of inversion center in the bulk GaAs, and the Rashba terms, which are caused by the asymmetry of the quantum well. It is shown that in $\mathrm{A}_{3} \mathrm{~B}_{5}$ quantum wells the magnetoresistance due to the weak localization depends qualitatively on the orientation of the well. In particular, it is demonstrated that the (110) geometry has a distinctive feature that in the absence of the Rashba terms the "antilocalization" effect, i.e. the positive magnetoresistance, does not exist. Calculation of the weak anti-localization magnetoresistance is found to be in excellent agreement with experiments.
\end{abstract}

\subsection{Fz,71.70.Ej,73.40.Kp,71.55.Eq}

\section{INTRODUCTION}

The effect of the negative magnetoresistance observed in high-density $2 d$ electron gas in semiconductor quantum wells is known to be caused by the weak localization, which results from the constructive interference of two electron waves propagating along a closed path in opposite directions, and leads to suppression of the conductivity. In a magnetic field the interference conditions are violated, which causes the effect of the negative magnetoresistance

It was shown in that the triplet states with the total momentum of both electron wavefunctions $J=1$ give a positive contribution into the resistance, while the singlet state with $J=0$ gives a negative contribution (antilocalization). Then, the interference conditions can also be changed by the spin relaxation, which, depending on the relaxation mechanism, can suppress the contribution of either triplet or (mainly) singlet statest. In the non-centrosymmetric semiconductors and semiconductor structures the dominant spin relaxation mechanism is the Dyakonov-Perel mechanism, whichis caused by the spin splitting of the conduction bandm. If this splitting is not very small, in weak magnetic fields the antilocalization effect prevails, and the resistance increases with the magnetic field $B$. Therefore, the nature and strength of the spin relaxation determines not just the magnitude of the negative magnetoresistance effect, but even the qualitative behavior of the magnetofgnductivity $\sigma(B)$. Furthermore, it was recently shownel that if the conduction band spin splitting is linear in the wave vector, which is always the case in $2 d$ structures, the theory of the weak localization must take into account the correlation between the electron motion in co-ordinate and spin spaces. The effects of the spin relaxation on the magnetoresistance were recently investigated experimentally

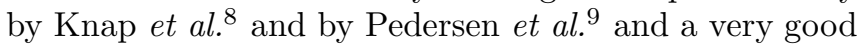
agreement was obtained with the theory for the (100) oriented GaAs quantum wells. In this paper we report for the first time on a study of magnetoresistance in (110)oriented quantum wells and present the theory of the weak localization for this particular case.

\section{THEORY}

In asymmetric $\mathrm{A}_{3} \mathrm{~B}_{5}$ quantum wells the spin splitting of the conduction band has two terms. The first, Dresselhaus term 10, arises from the asymmetry of the crystal itself and in the bulk crystal is described by the following Hamiltonian:

$$
\mathcal{H}_{1}=\gamma \sum \sigma_{i} k_{i}\left(k_{i+1}^{2}-k_{i+2}^{2}\right),
$$

where $i=x, y, z, i+3 \rightarrow i, \gamma$ is the spin-orbit coefficient for the bulk semiconductor, $\sigma_{i}$ are the Pauli matrices and $\vec{k}$ is the electron wave vector (in this paper we take $\hbar=1$ everywhere except in final formulas). We take the coordinate system $z\|110, x\| 1 \overline{1} 0$, and $y \| 001$. In a (110) quantum well the $k_{z}$ is quantized: $\left\langle k_{z}\right\rangle=0,\left\langle k_{z}^{2}\right\rangle=$ $\int|\nabla \psi|^{2} d z$, where $\psi(z)$ is the electron wave function in the well. Consequently, the Hamiltonian (1) becomes

$$
\mathcal{H}_{1}=-\gamma \sigma_{z} k_{x}\left[\frac{1}{2}\left\langle k_{z}^{2}\right\rangle-\frac{1}{2}\left(k_{x}^{2}-2 k_{y}^{2}\right)\right] .
$$

It is convenient to write this Hamiltonian as a sum of harmonics 6 䀚: 


$$
\mathcal{H}_{1}=\sigma_{z}\left(\Omega_{1 z}+\Omega_{3 z}\right)
$$

where

$$
\begin{aligned}
\Omega_{1 z} & =\Omega_{1} \cos \phi, \Omega_{3 z}=\Omega_{3} \cos 3 \phi, \\
\Omega_{1} & =-\frac{1}{2} \gamma k\left(\left\langle k_{z}^{2}\right\rangle-\frac{1}{4} k^{2}\right), \Omega_{3}=\frac{3}{8} \gamma k^{3}, \\
k^{2} & =k_{x}^{2}+k_{y}^{2}, \tan \phi=\frac{k_{y}}{k_{x}} .
\end{aligned}
$$

The other term in the conduction band spin splitting, the Rashba term, is caused by the asymmetry of the quantum well11. Its Hamiltonian does not depend on the orientation of the quantum well:

$$
\mathcal{H}_{2}=\left(\vec{\sigma} \cdot \vec{\Omega}_{2}\right),
$$

where $\Omega_{2_{x}}=\Omega_{2} \sin \phi, \Omega_{2_{y}}=-\Omega_{2} \cos \phi, \Omega_{2}=\alpha k$. In a uniform electric field $\mathcal{E}$ (triangular well) $\alpha=\alpha_{0} e \mathcal{E}$; the coefficient $\alpha_{0}$ may depend on the properties of the heterointerface.

Using the formalism similar to that of Refs. 68 one can show that the correction to the conductivity $\sigma$ caused by the weak localization is determined by the zero harmonic of the Cooperon $\mathbb{C}_{0}(\vec{q})$, which obeys the following equation:

$$
\mathcal{H} \mathbf{C}_{0}=\frac{1}{2 \pi \nu_{0} \tau_{0}^{2}}
$$

where $\nu_{0}$ is the density of states at the Fermi level, $\tau_{0}$ is the elastic lifetime and $v$ is the Fermi velocity. In the basis of the eigenfunctions $\phi_{0}$ (antisymmetric singlet state) and $\phi_{l}^{m}$ with $l=1, m=-1,0,1$ (symmetric triplet state) the operator $\mathcal{H}$ consists of two blocks, $\mathcal{H}_{0}$ for the singlet states and $\tilde{\mathcal{H}}$ for the triplet states:

$$
\begin{aligned}
\mathcal{H}_{0}= & D\left(q_{x}^{2}+q_{y}^{2}\right)+\frac{1}{\tau_{\varphi}}, \tilde{\mathcal{H}}=D\left(q_{x}^{2}+q_{y}^{2}\right)+\frac{1}{\tau_{\varphi}} \\
& +2\left[2 \Omega_{2}^{2}+J_{z}^{2}\left(\Omega_{1}^{2}-\Omega_{2}^{2}+\Omega_{3}^{2} \frac{\tau_{3}}{\tau_{1}}\right)-2 J_{y} J_{z} \Omega_{1} \Omega_{2}\right] \tau_{1} \\
& +2 v \tau_{1}\left[q_{x}\left(\Omega_{1} J_{z}-\Omega_{2} J_{y}\right)+q_{y} \Omega_{2} J_{x}\right] .
\end{aligned}
$$

Here $J_{i}$ are the matrices of the angular momentum operator with total momentum $J=1, \tau_{\varphi}$ is the phase-breaking time, $D=v^{2} \tau_{1} / 2$ is the diffusion coefficient, $\tau_{n}, n=1,3$, is the relaxation time of the respective component of the distribution function.

In a magnetic field $B \| z$ the wave vector $\mathbf{q}$ becomes an operator with the commutation relations

$$
\left[q_{+} q_{-}\right]=\frac{\delta}{D}, \delta=\frac{4 e B D}{\hbar c},
$$

where $q_{ \pm}=q_{x} \pm i q_{y}$.

This allows us to introduce creation and annihilation operators $a^{\dagger}$ and $a$, respectively, for which $\left[a a^{\dagger}\right]=1$ :

$$
D^{1 / 2} q_{+}=\delta^{1 / 2} a, \quad D^{1 / 2} q_{-}=\delta^{1 / 2} a^{\dagger}, \quad D q^{2}=\delta\left\{a a^{\dagger}\right\} .
$$

The weak localization correction to the conductivity in a magnetic field can now be written as

$$
\Delta \sigma=-\frac{e^{2} \delta}{4 \pi^{2} \hbar} \sum_{n=0}^{n_{\max }}\left(-\frac{1}{\mathcal{E}_{0 n}}+\sum_{m=-1}^{1} \frac{1}{E_{m n}}\right),
$$

where $n_{\text {max }}=1 / \delta \tau_{1}$. The eigenvalues $\mathcal{E}_{0 n}$ of $\mathcal{H}_{0}$ are given by the following equation:

$$
\mathcal{E}_{0 n}=\delta\left(n+\frac{1}{2}\right)+\frac{1}{\tau_{\varphi}} .
$$

The expression for the operator $\tilde{\mathcal{H}}$, of which $E_{m n}$ are the eigenvalues, follows from Eqs. (7, 9):

$$
\begin{aligned}
\tilde{\mathcal{H}}= & \delta\left\{a a^{\dagger}\right\}+\frac{1}{\tau_{\varphi}}+2\left(\Omega_{1}^{2} \tau_{1}+\Omega_{3}^{2} \tau_{3}\right) J_{z}^{2} \\
& +2\left(2-J_{z}^{2}\right) \Omega_{2}^{2} \tau_{1}-4 \Omega_{1} \Omega_{2} \tau_{1} J_{y} J_{z} \\
& +2\left(\delta \tau_{1}\right)^{1 / 2}\left[\frac{1}{\sqrt{2}} \Omega_{1} J_{z}\left(a^{\dagger}+a\right)+i \Omega_{2}\left(a^{\dagger} J_{+}-a J_{-}\right)\right] .
\end{aligned}
$$

where $J_{ \pm}=\left(J_{x} \pm i J_{y}\right) / \sqrt{2}$.

If we keep only the Dresselhaus terms in Eq. (7), i.e. put $\Omega_{2}=0$, the matrix $\tilde{\mathcal{H}}$ becomes diagonal in the basis of the eigenfunctions of $J_{z}$, and its non-zero matrix elements for arbitrary $n$ and $m=-1,0,1$ can be written as

$$
\tilde{\mathcal{H}}_{m m}=D\left[q_{y}^{2}+\left(q_{x}+q_{m}\right)^{2}\right]+2 \Omega_{3}^{2} \tau_{3} m^{2}+\frac{1}{\tau_{\varphi}},
$$

where $q_{m}=\left(2 \Omega_{1} / v\right) m$. Since the shift by $q_{m}$ does not change the commutation relations (8) for the operators $q_{y}$ and $q_{x}^{\prime} \equiv q_{x}+q_{m}$, the energies $E_{m n}$ depend only on the cubic Dresselhaus term:

$$
E_{m n}=\delta\left(n+\frac{1}{2}\right)+2 \Omega_{3}^{2} \tau_{3} m^{2}+\frac{1}{\tau_{\varphi}},
$$

while the spin relaxation rate is determined by the sum of all terms: $\tau_{s}^{-1}=2\left(\Omega_{1}^{2} \tau_{1}+\Omega_{3}^{2} \tau_{3}\right)$. One can see from Eqs. (10, 11, 14) that the term with $m=0$ cancels the contribution of $\mathcal{E}_{0 n}$ in the conductivity, and, therefore, the pragnetoconductivity $\Delta \sigma(B)$ is given by the expressiont:

$$
\begin{aligned}
& \Delta \sigma(B)-\Delta \sigma(0)= \\
& \frac{e^{2}}{2 \pi^{2} \hbar}\left\{\Psi\left(\frac{1}{2}+\frac{H_{\varphi}}{B}+\frac{H_{\mathrm{SO}}^{(3)}}{B}\right)-\ln \frac{H_{\varphi}}{B}\right\},
\end{aligned}
$$

where

$$
H_{\varphi}=\frac{c \hbar}{4 e D \tau_{\varphi}}, \quad H_{\mathrm{SO}}^{(3)}=\frac{c \hbar}{4 e D} 2 \Omega_{3}^{2} \tau_{3} .
$$


Therefore, in absence of the Rashba terms in a (110) quantum well the negative magnetoresistance cannot be observed.

When both Dresselhaus and Rashba terms are present, the eigenvalues of $\tilde{\mathcal{H}}$ can be found only numerically. In practice it is more convenient to compute directlythe sum of the inverse eigenvalues, using the expression 12 .

$$
\sum_{n} \sum_{m=-1}^{1} \frac{1}{E_{n m}}=\sum_{i} \frac{\left|D_{i i}\right|}{|D|}
$$

where $|D|$ is the determinant of the matrix $\tilde{\mathcal{H}}$ and $\left|D_{i i}\right|$ is the minor of its diagonal element $i, i$. The detailed description of the numerical procedure will be published elsewhere.

\section{EXPERIMENT}

The samples used in our work were grown by Molecular Beam Epitaxy (MBE) technique. The layer sequence was of the standard high mobility transistor type. The $2 d$ electron gas was formed in GaAs at the (110) GaAs $/ \mathrm{Ga}_{0.7} \mathrm{Al}_{0.3} \mathrm{As}$ interface. The sample was $\delta$-doped with silicon in two planes at $10 \mathrm{~nm}$ and $50 \mathrm{~nm}$ from the interface The individual samples were mesa-etched into rectangular Hall bars with the width of $0.2 \mathrm{~mm}$ and the total length of $4.2 \mathrm{~mm}$. 3 voltage contacts on each side were placed at a distance of $0.8 \mathrm{~mm}$ to avoid perturbing significantly the 4-point measurements. Ohmic contacts to the 2DEG was made by an annealed AuGeNiAu composite film in $0.6 \times 0.6 \mathrm{~mm}^{2}$ contact areas. The contacted areas were subsequently bonded to the legs of a non-magnetic chip carrier.

Our 4-point measurements of the resistivity were carried out by standard low-frequency lock-in technique. Typically the sample resistance was few $\mathrm{k} \Omega$ and with an AC current amplitude below $200 \mathrm{nA}$ we have avoided significant Joule heating of the sample at our lowest temperature, $0.3 \mathrm{~K}$. To generate low stable magnetic fields we used two highly stable current sources (Keithley 220); the first was used to out-compensate the magnetic flux trapped in the superconducting magnet, whereas the second was used for the magnetic field sweep around the zero value. The peak in the weak localization resistivity (or, for antilocalization, conductance) defines the zero value. Incidentally, this method is accurate enough to determine this zero point within about $1 \mu \mathrm{T}$ [3.

\section{RESULTS AND DISCUSSION}

In Fig. 1 we show the results of the magnetoconductivity measurements for a sample with electron density $n=5 \cdot 10^{11} \mathrm{~cm}^{-2}$ and mobility $\mu=7 \cdot 10^{4} \mathrm{~cm}^{2} / \mathrm{Vs}$ at $T=0.36 \mathrm{~K}$. Also shown are the best fits as obtained from our theory, and from the theory of Hikami, Larkin,

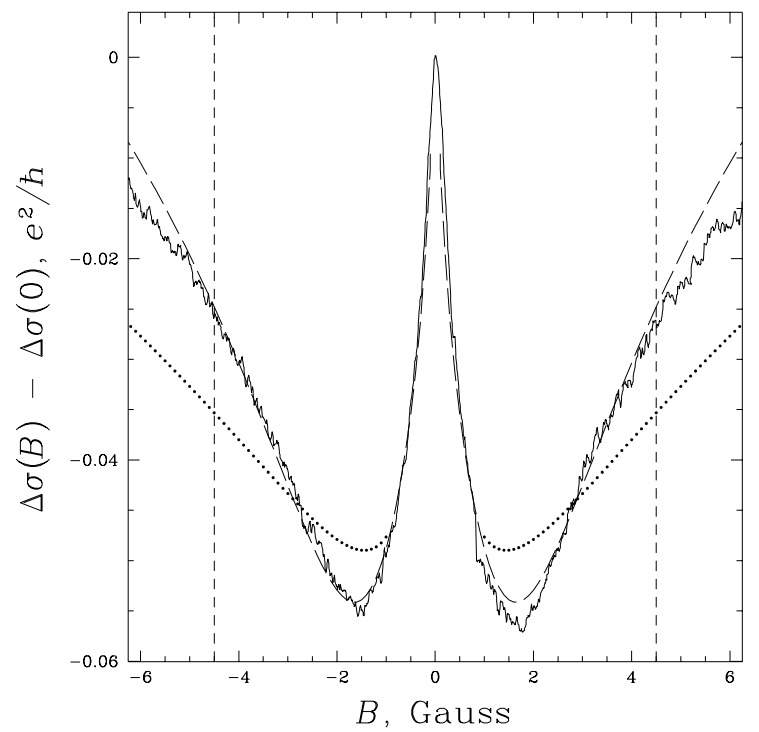

FIG. 1. Magnetoconductivity $\Delta \sigma(B)-\Delta \sigma(0)$ in (110) quantum well. Experimental results are shown by solid line, theoretical best fit - by dashed line The dots show the best fit by the Hikami-Larkin-Nagaoka theory. Sample characteristics and parameters of the theory are given in the text. The vertical lines show the interval $|B| \leq H_{\mathrm{tr}}=4.5$ Gs.

and Nagaoka (HLN)2, which assumes that all terms of the spin splitting give additive contributions into magnetoconductivity. The fitting was done by weighted explicit orthogonal distance regression using the software package ODRPACK14. The weights were selected to increase the importance of the low-field ( $B \leq 3 \mathrm{Gs})$ part of the magnetoconductivity curve. Only the experimental points at $|B| \leq H_{\mathrm{tr}}=\frac{c \hbar}{4 e D \tau_{1}}=4.5 \mathrm{Gs}$ we used for fitting, since the above theories use the diffusion approximation, and, therefore, are only valid for $B$ small compared to $H_{\mathrm{tr}}$.

The parameters of our theory are $\tau_{\varphi}$ and $\Omega_{i}, i=1,2,3$. It is convenient to convert them into characteristic magnetic fields $H_{\varphi}, H_{\mathrm{SO}}^{(3)}$ ( see Eq. (16)), and $H_{\mathrm{SO}}^{(1,2)}$ :

$$
H_{\mathrm{SO}}^{(1)}=\frac{c \hbar}{4 e D} 2 \Omega_{1}^{2} \tau_{1}, H_{\mathrm{SO}}^{(2)}=\frac{c \hbar}{4 e D} 2 \Omega_{2}^{2} \tau_{1} .
$$

The parameters of the best fit are $H_{\varphi}=0.02 \mathrm{Gs}, H_{\mathrm{SO}}^{(1)}=$ $0.12 \mathrm{Gs}, H_{\mathrm{SO}}^{(2)}=1.3 \mathrm{Gs}$, and $H_{\mathrm{SO}}^{(3)}=0.04 \mathrm{Gs}$ for our theory and $H_{\varphi}=0.014 \mathrm{Gs}, H_{\mathrm{SO}}=0.33$ Gs for the HLN theory. One can clearly see that the HLN theory is unable to describe the experimental data. The disagreement between the experiment and the HLN theory in ourcase is much more severe than for (001) quantum wells 7 , since the effects of the correlations between the electron motion in co-ordinate and spin spaces is much stronger here: as we have shown above, in a (110) quantum well the linear Dresselhaus terms have no effect on the magnetoconductivity (in the absence of the Rashba term), whereas in a (001) well such a dramatic cancellation is only possible when both Rashba and Dresselhaus terms exist and are nearly equal. 
From the above values of the parameters $H_{\mathrm{SO}}^{(i)}$ we can determine the values of the constants $\gamma$ and $\alpha_{0}$, using Eqs. (41, 16, 18), $k_{\mathrm{F}}=\sqrt{2 \pi N_{\mathrm{s}}}$, and the following expressions for $\left\langle k_{z}^{2}\right\rangle 15$ and $\alpha$, which are obtained using the standard variational wavefunction for electrons at the heterointerface 16 :

$$
\left\langle k_{z}^{2}\right\rangle=\frac{1}{4}\left(\frac{16.5 \pi e^{2} m N_{\mathrm{s}}}{\kappa \hbar^{2}}\right)^{1 / 3}, \alpha=e \overline{\mathcal{E}} \alpha_{0}, \overline{\mathcal{E}}=\frac{2 \pi e N_{\mathrm{s}}}{\kappa},
$$

where $N_{\mathrm{s}}$ is the electron density, $\kappa$ is the dielectric constant, $m$ is the effective electron mass, and $\overline{\mathcal{E}}$ is the average electric field in the well. The resulting values of the coefficients are $\gamma \approx 22 \mathrm{eV} \AA^{3}$ and $\alpha_{0} \approx 14 \AA^{2}$. The value of $\gamma$ is very close to the presioms reported values, both

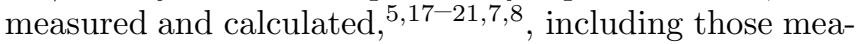
surecin weak localization experiments in (001) quantum wells 6 . The value of $\alpha_{0}$ was only measured in the latter experiments and reported to be about $7.2 \AA^{2}$. However, unlike $\gamma$, which is the bulk material coefficient, $\alpha_{0}$ may contributions from the interface 22 23. Their magnitude is not reliably known; therefore, we do not view the discrepancy between our value of $\alpha_{0}$ and the one measured in (001) wells as alarming. Also, from the value of $H_{\text {SO }}^{(3)}$ we can determine the ratio $\tau_{3} / \tau_{1} \approx 1 / 8$. This ratio can vary from 1 for short-range scattering to $1 / 9$ for scattering on remote impurities, and the experiment shows that in our samples those are practically the only source of scattering. Lastly, we can determine the phase relaxation time $\tau_{\varphi} \approx 6 \cdot 10^{-10} \mathrm{~s}$.

\section{CONCLUSIONS}

In conclusion, we have presented new experimental and theoretical studies of magnetoconductivity caused by the weak localization in (110) GaAs quantum wells. It is demonstrated that if the spin splitting of the conduction band is linear in the wave vector it is necessary to take into account the correlation between the electron motion in co-ordinate and spin spaces. This correlation leads to the special feature of the (110) geometry: in a perfectly symmetric quantum well, when the Rashba terms are absent, the weak antilocalization effect, which leads to positive magnetoresistance, does not exist. The presence of the positive magnetoresistance in our samples is a clear signature of the Rashba terms in the conduction band spin splitting. Our new theory achieves a good agreement with the experiment and gives the values for the parameters of the spin splitting which are in agreement with previous optical and transport experiments and theoretical calculations.

We thank W. Knap communicating preprints prior to publication. The experimental part of our research was carried out at NBIfAFGs III-V NANOLAB and supported by the Center for Nanostructures (CNAST) under the MUP II program. F. G. P. acknowledges support by the NSF Grant DMR 93-08011, the Center for Quantized Electronic Structures (QUEST) of UCSB and by the Quantum Institute of UCSB. G. E. P. acknowledges support by RFFI Grant 96-02-17849 and by the Volkswagen Foundation.

$\dagger$ New address: Department of Physics, State University of New York at Stony Brook, Stony Brook, NY 11794, USA.

${ }^{1}$ B. Altshuler, D. Khmelnitskii, A. Larkin and P. Lee, Phys. Rev. B 22, 5142 (1980).

${ }^{2}$ S. Hikami, A. Larkin, and Y. Nagaoka. Progr. Theor. Phys. 63, 707 (1980).

${ }^{3}$ B. L. Altshuler, A. G. Aronov, A. I. Larkin, and D. E. Khmelnitskii, JETP 54, 411 (1981) (JETP 81, 788 (1981)).

${ }^{4}$ M. I. Dyakonov and V. I. Perel, JETP 33, 1053 (1971).

${ }^{5}$ G. E. Pikus and A. Titkov, "Spin Relaxation under Optical orientation", in "Optical Orientation", ed. by F. Mayer and B. Zakharchenya, North Holland, Amsterdam (1984).

${ }^{6}$ S. V. Iordanskii, Yu. B. Lyanda-Geller, and G. E. Pikus, JETP Letters 60, 206 (1994) (Pisma v JETP 60, 199 (1994)).

${ }^{7}$ F. G. Pikus and G. E. Pikus, Phys. Rev. B. 51, 16928 (1995).

${ }^{8}$ W. Knap et. al, Phys. Rev. B. 53, 3912 (1996).

${ }^{9}$ S. Pedersen, T. Hassenkam, and P. E. Lindelof, Czech. J. Phys. LT21, to be published (1996).

${ }^{10}$ G. Dresselhaus, Phys. Rev. 100, 580 (1955).

${ }^{11}$ E. I. Rashba and V. I. Sheka, Fiz. Tverd. Tela, Sb. II, 162(1959); E. I. Rashba and V. I. Sheka, Sov. Phys. Sold State, 3, 1257 (1961).

${ }^{12}$ F. G. Pikus and G. E. Pikus, Solid State Commun., in press.

${ }^{13}$ F. B. Rasmussen, et al., Czech. J. Phys. LT21, to be published.

${ }^{14}$ P. T. Boggs et. al, NIST preprint NISTIR 92-4834.

${ }^{15}$ T. Ando, A. B. Fowler, and F. Stern, Rev. Mod. Phys., 54, 437 (1982).

${ }^{16}$ F. F. Fang and W. E. Howard, Phys. Rev. Lett., 16, 797 (1966).

${ }^{17}$ P. Vögl, H. P. Hjalmarson, and J. D. Dow, J. Chem. Solids, 44, 365 (1983).

${ }^{18}$ A. Kobayashi, O. F. Sankey and J. D. Dow, Phys. Rev. B 25, 6367 (1982).

${ }^{19}$ P. V. Santos and M. Cardona, Phys. Rev. Lett. 72, 3 (1994).

${ }^{20}$ P. V. Santos, M. Willatzen, M. Cardona, and A. Santarero, Phys. Rev. B 51, 121 (1995).

${ }^{21}$ B. Jusserand, D. Richards, G. Allan, C. Priester, and B. Etienne, Phys. Rev. B. 51, 4707 (1995).

${ }^{22}$ L. G. Gerchikov and A. W. Subashiev, Sov. Phys. Semicond. 26, 73 (1992) (Fiz. Techn. Poluprov. 26, 131 (1992)).

${ }^{23}$ G. E. Pikus and U. Rössler, unpublished. 